\title{
Fetomaternal microchimerism
}

\section{Some answers and many new questions}

Kian Hwa Tan, Xiao Xia Zeng, Piriya Sasajala, Ailing Yeo and Gerald Udolph*

Institute of Medical Biology; Singapore

Key words: fetomaternal microchimerism, fetal progenitor cells, differentiation, pregnancy associated progenitor cells, organ specific integration

Submitted: $12 / 02 / 10$

Accepted: 12/20/10

DOI: $10.4161 /$ chim.2.1.14692

*Correspondence to: Gerald Udolph;

Email: gerald.udolph@imb.a-star.edu.sg

Addendum to: Zeng XX, Tan KH, Yeo A, Sasajala P, Tan X, Xiao ZC, et al. Pregnancy-associated progenitor cells differentiate and mature into neurons in the maternal brain. Stem Cells Dev 2010; 19:1819-30; PMID: 20707697; DOI: 10.1089/ scd.2010.0046.
$\mathrm{T}$ he transfer of fetal cells into mothers during pregnancy and their organ specific integration is a well recognized phenomenon in placental vertebrates. Recently, it has been reported that some fetal cells found in the mothers have progenitor cell-like features such as multilineage differentiation potential and as a consequence they were termed pregnancy associated progenitor cells (PAPC). The multilineage differentiation potential suggested some level of cellular plasticity, which these cells share with other stem or progenitor cells. In this context, we have shown that PAPCs indeed express neural stem cell and markers for developing neurons in the brain and that PAPCs morphologically mature into neurons over time. The stem/progenitor properties of PAPCs raises the hope that they might be valuable for studying the functional integration of foreign cells into preexisting tissues and organs, for example in cellular therapies. The functional integration of transplanted cells and their connectivity to the host circuitry is still a major bottleneck in cellular therapies particularly for the brain. The animal models of fetomaternal microchimerism might provide valuable insights into the mechanism how cells survive, migrate, integrate and differentiate in a foreign environment of a host. This review discusses some of the recent findings in the field of fetomaternal microchimerism. It also tries to identify some major gaps of knowledge and raises some questions resulting from the recent advances. Studying fetomaternal microchimerism and the properties of PAPCs in greater detail might pave the way to advance cell based regenerative medicine as well as transplantation medicine.

Fetomaternal microchimerism (FMc) is a special form of chimerism observed in placental vertebrates in whom a small number of fetal cells called PAPCs migrate into the mother and integrate into maternal organs during pregnancy. In humans, PAPCs have been described to persist in mothers almost three decades postpartum $^{1}$ suggesting that they have long-term survival and engraftment capabilities. The phenomenon of cell migration between fetus and mother during pregnancy has already been described as early as in the 1950s in humans as well as in vertebrate animal models such as the mouse. ${ }^{2-5}$ From a clinical perspective, fetal cells circulating in the mothers were initially promising as a less invasive approach for prenatal diagnostics. ${ }^{6-8}$ Furthermore, the presence of fetal cells was also hypothesized to contribute to pregnancies-associated disorders such as autoimmune diseases. ${ }^{9-12}$ Recent studies of FMc in animal models have been of great value as they have provided fundamental novel insights into the developmental capacity/plasticity of these fetal cells. Lately, reports have emerged that some PAPCs possessed features of progenitor cells. ${ }^{13,14}$ PAPCs have been reported to differentiate into a multitude of different cell types in maternal organs such as in the blood, ${ }^{15}$ the $\operatorname{skin}^{16-18}$ and in the central nervous system. ${ }^{19,20}$ PAPCs were also found in the liver and kidney where they participated in tissue regeneration after injury. ${ }^{21,22}$ Despite the expression of tissue specific cellular phenotypes, the mechanism for functional integration of 


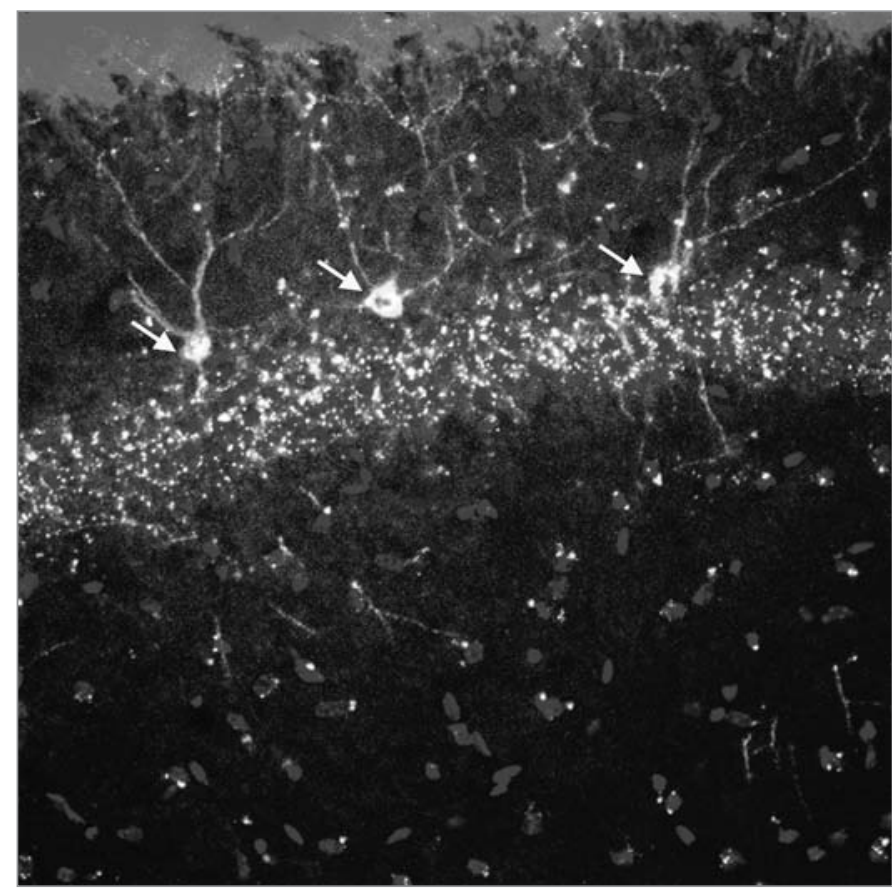

Figure 1. Three PAPCs are shown (yellow, white arrows) coexpressing the fetal cell marker GFP and the neuronal marker $\beta-3$ tubulin in the cellular layer of the hippocampus of a murine mother. Note that PAPCs show axonal and dendritic aborizations, which are comparable to those from endogenous maternal hippocampal neurons. This indicates that PAPCs adopted a site specific identity.

PAPCs into most of the studied organs still requires further extensive research.

Our group has recently shown that PAPCs with a neuronal phenotype can be detected in maternal mice up to seven months postpartum (Fig. 1). ${ }^{20}$ This demonstrated that fetal cells can persist longterm in the brain as neurons in many different brain regions, potentially for the life time of a mouse. Acquisition of neuronal cell fate was not the consequence of cell fusion but PAPCs differentiated intrinsically which probably involved a gradual process from a stem/progenitor like cell type via immature neurons to mature neurons. Furthermore, the patterns of marker gene expression in PAPCs were very reminiscent of stage specific marker gene expression described for adult neural stem cells and their differentiation. ${ }^{23}$ This suggested that PAPCs in the brain exhibited some features of typical neural stem cells.

A major focus of interest recently has been to elucidate mechanisms of PAPC migration and attraction to particular sites in the maternal body. To this end, injury and cancer models have been used to investigate targeted migration of these cells and it was found that injury ${ }^{16-20,22}$ as well as cancer ${ }^{24,25}$ attracted PAPCs. Although not much is known about the underlying mechanisms, it can be speculated that cytokine cytokine-receptor systems might be involved in fetal cell migration and attraction. However, specific mechanisms attracting fetal cell types to different maternal tissues, particularly to non-injured tissues have yet to be identified. Along their journey, e.g., to the brain, PAPCs have to cross at least two major endothelial barriers, the placental as well as the blood-brain barrier. The mechanisms facilitating endothelial transmigration are not understood. Although it could well be that PAPC migration is stochastic and does not require any specific signaling, further studies are needed to decipher migratory properties of PAPCs and possible mechanisms involved.

The major and currently the most limiting factor is the notoriously low frequency of detectable PAPCs in mothers. However, some improvement has been made by increasing the number of PAPCs through experimental break-down of the placental barrier. ${ }^{26}$ These experiments, however, did not address whether the extra cells are true PAPCs with multilineage differentiation potential or whether at least in part they are an influx of non-PAPC cells as a consequence of the break-down of a functional placental barrier. But generally any approach to increase the frequency of PAPCs in mothers has the potential to enhance our understanding of FMc.

Although a lot has been learned about the potential of PAPCs in terms of their homing, survival and differentiation into different organ specific cell types, much more needs to be learned about the origin as well as the identity of PAPCs. As it currently stands, the definition of PAPCs is operational and is simply based on the presence of fetal cells, sometimes differentiated, in the mother but does not include any clear functional criteria for a defined population of cells. Of foremost interest in this context is also the question whether PAPCS are a unique type of stem or progenitor cell with multilineage differentiation potential or whether different progenitor cells with limited differentiation potential migrate, home and differentiate into specific sets of organs in the mother. A major challenge here could be that PAPCs might be a moving target since it may be difficult to determine whether a cell of fetal origin is still in its immature stage when found in the mother or whether it has undergone some level of differentiation already. Genetic tracking experiments of specific fetal cell populations might provide the required insights. Currently, however, neither the unique stem cell nor the limited progenitor cell hypothesis can be verified by the available data.

Furthermore, for PAPCs to truly move towards possible clinical applications, the isolation as well as propagation of PAPCs in vitro with multilineage potential has yet to be mastered. This is of utmost importance, firstly for performing the necessary in vitro studies addressing questions regarding the basic biology of PAPCs, e.g., their differentiation potential, and for achieving clinically relevant numbers of transplantable cells by scaled-up in-vitro production of PAPCs.

Another striking feature of PAPCs in the human system is the long-term 
persistence of foreign cells in mothers that requires some level of immune tolerance by the mother or a mechanism, which allows PAPCs to evade an attack by the maternal immune system. The mechanisms of immune evasion could be of critical importance as PAPCs could teach us the trick of how cells could escape any immune response by the host. If such escape properties are a cardinal feature of PAPCs that is maintained also in differentiated PAPCs this, in combination with their multilineage differentiation potential, might be of clinical relevance.

In summary, it has become evident recently that PAPCs seem to share some common features with stem or progenitor cells at least with regard to the differentiation potential although self-renewal capacity of PAPCs has not been demonstrated yet. This feature provides some hope that PAPCs could be of clinical significance for regenerative medicine. Also a lot can be learned from PAPCs that might be of relevance for cellular therapies as their transfer to mothers mimics an allogenic transplantation paradigm. Although fetomaternal microchimerism is intriguing and persistent microchimerism was unexpected, a major question will be whether or not fetomaternal microchimerism is a biological accident happening during pregnancy without any relevance to fetus and mother, or whether there is yet unknown biological significance to it. It also remains to be seen whether PAPCs are capable of revolutionizing stem cell therapy approaches in the future.

\section{References}

1. Bianchi DW, Zickwolf GK, Weil GJ, Sylvester S, DeMaria MA. Male fetal progenitor cells persist in maternal blood for as long as 27 years postpartum. Proc Natl Acad Sci USA 1996; 93:705-58.

2. Greger WP, Steele MR. Human fetomaternal passage of erythrocytes. N Engl J Med 1957; 256:158-61.

3. Desai RG, Creger WP. Maternofetal passage of leukocytes and platelets in man. Blood 1963; 21:665-73.

4. Lee RE, Vazquez JJ. Immunocytochemical evidence for transplacental passage of erythrocytes. Lab Invest 1962; 11:580-4.

5. Tuffrey M, Bishun NP, Barnes RD. Porosity of the mouse placenta to maternal cells. Nature 1969; 221:1029-30

6. Bianchi DW. Prenatal diagnosis by analysis of fetal cells in maternal blood. J Pediatr 1995; 127:847-56.

7. de la Cruz F, Shifrin H, Elias S, Simpson JL, Jackson $\mathrm{L}$, Klinger K, et al. Prenatal diagnosis by use of fetal cells isolated from maternal blood. Am J Obstet Gynecol 1995; 173:1354-5.

8. Bianchi DW. Fetal cells in the maternal circulation feasibility for prenatal diagnosis. Br J Haematol 1999; 105:574-83.

9. Nelson JL. Maternal-fetal immunology and autoimmune disease: is some autoimmune disease autoalloimmune or allo-autoimmune? Arthritis Rheu Feb 1996; 39:191-4.

10. Nelson JL. Autoimmune disease and the long-term persistence of fetal and maternal microchimerism. Lupus 1999; 8:493-6.

11. Nelson JL. Microchimerism in human health and disease. Autoimmunity 2003; 36:5-9.

12. Bianchi DW. Fetomaternal cell traffic, pregnancyassociated progenitor cells and autoimmune disease. Best Pract Res Clin Obstet Gynaecol 2004; 18:959-75.

13. Khosrotehrani K, Bianchi DW. Multi-lineage potential of fetal cells in maternal tissue: a legacy in reverse. J Cell Sci 2005; 118:1559-63.

14. Bianchi DW, Robert E. Gross Lecture. Fetomaternal cell trafficking: a story that begins with prenatal diagnosis and may end with stem cell therapy. J Pediatr Surg 2007; 42:12-8

15. Khosrotehrani K, Leduc M, Bachy V, Nguyen Huu S, Oster M, Abbas A, et al. Pregnancy allows the transfer and differentiation of fetal lymphoid progenitors into functional $\mathrm{T}$ and $\mathrm{B}$ cells in mothers. J Immunol 2008; 180:889-97.
16. Nguyen Huu S, Oster M, Uzan S, Chareyre F, Aractingi S, Khosrotehrani K. Maternal neoangiogenesis during pregnancy partly derives from fetal endothelial progenitor cells. Proc Natl Acad Sci USA 2007; 104:1871-6.

17. Nguyen Huu S, Khosrotehrani K, Oster M, Moguelet P, Espie MJ, Aractingi S. Early phase of maternal skin carcinogenesis recruits long-term engrafted fetal cells. Int J Cancer 2008; 123:2512-7.

18. Nguyen Huu S, Oster M, Avril MF, Boitier F, Mortier L, Richard MA, et al. Fetal microchimeric cells participate in tumour angiogenesis in melanomas occurring during pregnancy. Am J Pathol 2009; 174:630-7.

19. Tan XW, Liao H, Sun L, Okabe M, Xiao ZC, Dawe GS. Fetal microchimerism in the maternal mouse brain: a novel population of fetal progenitor or stem cells able to cross the blood-brain barrier? Stem Cells 2005; 23:1443-52.

20. Zeng XX, Tan KH, Yeo A, Zeng XX, Tan KH, Yeo A, et al. Pregnancy-associated progenitor cells differentiate and mature into neurons in the maternal brain. Stem Cells Dev 2010; 19:1819-30.

21. Wang Y, Iwatani H, Ito T, Horimoto N, Yamato M, Matsui I, et al. Fetal cells in mother rats contribute to the remodeling of liver and kidney after injury. Biochem Biophys Res Commun 2004; 325:961-7.

22. Khosrotehrani K, Reyes RR, Johnson KL, Freeman RB, Salomon RN, Peter I, et al. Fetal cells participate over time in the response to specific types of murine maternal hepatic injury. Hum Reprod 2007; 22:654-61.

23. Kempermann G, Jessberger S, Steiner B, Kronenberg G. Milestones of neuronal development in the adult hippocampus. Trends Neurosci 2004; 27:447-52.

24. Dubernard G, Aractingi S, Oster M, Rouzier R, Mathieu MC, Uzan S, et al. Breast cancer stroma frequently recruits fetal derived cells during pregnancy. Breast Cancer Res 2008; 10:14.

25. Dubernard G, Oster M, Chareyre F, Antoine M, Rouzier R, Uzan S, et al. Increased fetal cell microchimerism in high grade breast carcinomas occurring during pregnancy. Int J Cancer 2009; 124:1054-9.

26. Johnson KL, Tao K, Stroh H, Kallenbach L, Peter I, Richey L, et al. Increased fetal cell trafficking in murine lung following complete pregnancy loss from exposure to lipopolysaccharide. Fertil Steril 2010; 93:1718-21. 